\title{
Exploration of microRNA profiles in human colostrum
}

\author{
Fei $\mathrm{Wu}^{1,2 \#}$, Xinyue Zhi ${ }^{1,2 \#}$, Rong Xu ${ }^{1}$, Zhiyi Liang ${ }^{3}$, Fang $\mathrm{Wang}^{4}$, Xiaoyu $\mathrm{Li}^{1}$, Yongmei $\mathrm{Li}^{5}$, Bei Sun ${ }^{1 \wedge}$ \\ ${ }^{1}$ NHC Key Laboratory of Hormones and Development, Tianjin Key Laboratory of Metabolic Diseases, Chu Hsien-I Memorial Hospital \& \\ Tianjin Institute of Endocrinology, Tianjin Medical University, Tianjin, China; ${ }^{2}$ Department of Epidemiology and Biostatistics, School of Public \\ Health, Tianjin Medical University, Tianjin, China; ${ }^{3}$ Department of Physiology and Pathophysiology, Collaborative Innovation Center of Tianjin for \\ Medical Epigenetics, Tianjin Medical University, Tianjin, China; ${ }^{4}$ The Second Hospital of Tianjin Medical University, Tianjin, China; ${ }^{5}$ Department \\ of Pathogenic Biology, School of Basic Medical Sciences, Tianjin Medical University, Tianjin, China \\ Contributions: (I) Conception and design: B Sun, Y Li; (II) Administrative support: B Sun, Y Li; (III) Provision of study materials or patients: Z Liang, \\ F Wang; (IV) Collection and assembly of data: X Zhi, F Wu; (V) Data analysis and interpretation: X Li, R Xu; (VI) Manuscript writing: All authors; \\ (VII) Final approval of manuscript: All authors. \\ \#These authors contributed equally to this work. \\ Correspondence to: Bei Sun, MD, PhD. Key Laboratory of Hormones and Development (Ministry of Health), Tianjin Key Lab of Metabolic Diseases, \\ Tianjin Metabolic Diseases Hospital \& Institute of Endocrinology, Tianjin Medical University, Tianjin, China. Email: sun_peipei220@hotmail.com; \\ Yongmei Li, PhD. Department of Pathogen Biology, School of Basic Medical Sciences, Tianjin Medical University, Tianjin, China. \\ Email: liym@tmu.edu.cn.
}

Background: Colostrum is well known to have excellent nutritional value for newborns. The aim of this study was to investigate the dynamic expression pattern of microRNA in human colostrum and mature milk. Furthermore, we identified the specific microRNA in human colostrum and analyzed the regulatory function of human colostrum.

Methods: We collected breast milk samples from 18 lactating volunteers. The expression of microRNA in breast milk was detected by microarray analysis. The expression differences were characterized by $\log 2 \mathrm{FC}$ ( $\mid \log 2$ fold change $\mathrm{I}>1.58)$ and associated $\mathrm{P}$ values $(\mathrm{P}<0.05)$. Furthermore, the prediction of microRNA targets, bioinformatics analysis and network generation were carried out using network database.

Results: Our results showed that during the human lactation process, the composition of microRNAs in human milk changes dynamically. Compared to the microRNA expression profile in human mature milk, the expression levels of 49 microRNAs were significantly different and 67 microRNAs were specifically expressed in human colostrum. Based on the results of Gene Ontology (GO) and Kyoto Encyclopedia of Genes and Genomes (KEGG) pathway enrichment analysis, the predicted target mRNAs of the identified colostrum-specific microRNAs were involved in the regulation of distinct biological processes, such as signal transduction, positive regulation of GTPase activity, and protein phosphorylation. Moreover, the predicted mRNA targets were from large spectrums of signaling pathways, such as the MAPK, Ras, Hippo, Wnt, and mTOR signaling pathways, as well as the longevity regulating pathway.

Conclusions: Our study illuminates the landscape of microRNA expressions in human colostrum and mature milk, and emphasizes the value of microRNAs as nutritional additives in milk-related commercial products.

Keywords: MicroRNAs (miRNAs); colostrum; mature milk; microarray

Submitted Jul 16, 2020. Accepted for publication Sep 01, 2020.

doi: 10.21037/atm-20-5709

View this article at: http://dx.doi.org/10.21037/atm-20-5709

^ ORCID: 0000-0003-0897-8831. 


\section{Introduction}

Breastfeeding has many nutritional, immune, physical, developmental, and psychological benefits, which makes human breast milk the best food for newborns, as well as the most natural. A large prospective cohort study in Brazil reported the total duration of breastfeeding and predominant breastfeeding to be positively associated with intelligence quotient, educational attainment, and income (1). The World Health Organization recommends that infants should be exclusively breastfed for the first 6 months of life. Human breast milk contains at least 415 proteins. These proteins are involved in all aspects of metabolism and various functions of the body (2). Colostrum contains more nutrients than mature milk, making it more beneficial for babies.

MicroRNAs (miRNAs) are endogenous non-coding RNAs of 18-25 nt in length. They are important regulators of many biological processes, such as organogenesis, body development, cellular metabolism, and cell differentiation (3). Moreover, miRNAs have unique stable expression patterns in various body fluids, including saliva (4), serum (5,6), plasma (7), and urine (8). Therefore, miRNAs can be used as non-invasive or minimally invasive biomarkers for a range of diseases, as well as potential indicators for the quality of food products (9). Exogenous miRNAs derived from diet can regulate the expressions of target genes and be used to identify specific tissue cells (10). MiR-168a, for instance, is abundant in rice and among the most highly enriched exogenous plant miRNAs in sera of Chinese populations (11). Previous functional studies have demonstrated that miR-168a binds to the mRNA of human/ mouse low-density lipoprotein receptor adapter protein 1 and inhibits its protein expression in the liver; consequently, causing impairment in removal of low-density lipoprotein from plasma $(10,11)$.

Exogenous miRNAs are stable in the blood of mammals. In recent years, studies on the functional properties and potential applications of miRNAs in the food field have increased. MiRNAs are involved in mammary gland development and milk ingredient synthesis (12). MiRNAs also comprise the nutritive indexes of fluid milk and powdered-formula milk (13). To date, the unique miRNA expression profiles in human colostrum and mature milk have rarely been reported. In the current study, we aimed to investigate dynamic miRNA expression patterns in human colostrum compared with mature milk in order to identify miRNAs specifically expressed in human colostrum. For the first time, this study shines a light on miRNA expression in human colostrum and mature milk, and emphasizes the value of miRNAs as nutritional additives in milk-related commercial products.

We present the following article in accordance with the MDAR reporting checklist (available at http://dx.doi. org/10.21037/atm-20-5709).

\section{Methods}

\section{Sample collection}

Human milk samples were collected from 18 lactating volunteers (age $30 \pm 4 \mathrm{y}$, pregnancy $40 \pm 3 \mathrm{w}$ ). Breast milk produced on days 7 and 14 after birth is defined as transition milk. Previous reports have clearly shown that nutritional compositions of breast milk vary throughout lactation period $(14,15)$. Colostrum is rich in growth factors and immunologic compounds (16), whereas components in mature milk is most likely stable without further fluctuations (17). The Nutritional components of colostrum are obviously different from those of mature milk. Therefore, we collected samples of colostrum (17 days) and mature milk (14 days) to perform our analysis. All human milk samples $(\mathrm{n}=18,8$ colostrum samples and 10 mature milk samples), were expressed by hand between 8 a.m. and 11 a.m., and stored at $-80^{\circ} \mathrm{C}$. Two colostrum and two mature milk samples were subjected to microarray analysis. All participants were Han Chinese from Tianjin, China, and were healthy, with no hypertension, diabetes, or other diseases. None of the participants were smokers or drinkers, and none of them were pregnant. All procedures performed in this study involving human participants were in accordance with the Declaration of Helsinki (as revised in 2013). This study was approved by Ethics Committee of the second hospital of Tianjin Medical University (No. KY2020K055) and informed consent was taken from all the patients.

\section{MiRNA functional enrichment analyses}

Microarray analyses were performed at Shanghai Biotechnology Corporation (Shanghai, China), using Agilent SurePrint Human miRNA 8×60 K V21.0 microarray (Agilent Technologies, USA). Expression differences were characterized by $\log _{2} \mathrm{FC}\left(\mid \log _{2}\right.$ fold change $\left.\mathrm{I}>1.58\right)$ and $\mathrm{P}$ values $(\mathrm{P}<0.05)$. The analyses were performed using the $\mathrm{R}$ package of edgeR (18). 
Table 1 Primer sequences for quantitative RT-PCR

\begin{tabular}{|c|c|}
\hline Gene name & Primer sequences $\left(5^{\prime}-3^{\prime}\right)$ \\
\hline hsa-miR-623 & Forward: GGTCCATCCCTTGCAGGGGCTG \\
\hline \multirow[t]{2}{*}{ hsa-miR-885-5p } & Stemloop: GTCGTATCCAGTGCAGGGTCCGAGGTATTCGCACTGGATACGACAGAGGC \\
\hline & Forward: GGTCCTCCATTACACTACCCTG \\
\hline hsa-miR-429 & Forward: GGTCCTAATACTGTCTGGTAAA \\
\hline \multirow[t]{2}{*}{ hsa-miR-511-3p } & Stemloop: GTCGTATCCAGTGCAGGGTCCGAGGTATTCGCACTGGATACGACTCTGTC \\
\hline & Forward: GGTCCGTGTAGCAAAAGACAGA \\
\hline hsa-miR-29c-3p & Stemloop: GTCGTATCCAGTGCAGGGTCCGAGGTATTCGCACTGGATACGACTAACCG \\
\hline hsa-miR-183-5p & Forward: GGTCCAAGATGGTCACGGTAT \\
\hline \multirow[t]{2}{*}{ hsa-miR-30b-5p } & Stemloop: GTCGTATCCAGTGCAGGGTCCGAGGTATTCGCACTGGATACGACAGCTGA \\
\hline & Forward: GGTCCATCCTACACTCAGCT \\
\hline \multirow[t]{2}{*}{ cel-miR-39 } & Stemloop: GTCGTATCCAGTGCAGGGTCCGAGGTATTCGCACTGGATACGACCAAGCT \\
\hline & Forward: GGTCCGTGTAAATCAGCTTG \\
\hline Universal reverse & CCAGTGCAGGGTCCGAGGT \\
\hline
\end{tabular}

RT-PCR, real-time polymerase chain reaction.

Validation of candidate miRNAs by quantitative real-time polymerase chain reaction ( $q R T-P C R$ )

Equal volumes of the milk samples were centrifuged at $2000 \mathrm{~g}$ for 10 minutes at $4{ }^{\circ} \mathrm{C}$. Then, the same volume of Caenorbabditis elegans miR-39 (cel-miR-39, UCACCGGGUGUAAAUCAGCUUG) was spiked into each milk samples as internal reference for normalization (19). MiRNAs were extracted from the milk samples using EasyPure ${ }^{\circledR}$ miRNA Kit (Transgen Biotech, China) and detected by RT-PCR with SGExcel Fast SYBR Mixture (Sangon Biotech, Shanghai, China), according to the manufacturer's protocol. Each sample was analyzed in triplicate. The miRNA primer sequences were synthesized by Tsingke Biotech (Beijin, China) according to the miRBase database (Release 20.0) and are listed in Table 1. The expression levels of miRNAs were calculated using the $2^{-\Delta \Delta \mathrm{Ct}}$ method.

\section{Prediction of miRNA targets, bioinformatic analysis, and network generation}

The target genes of the differentially expressed miRNAs were predicted using miRDB (http://mirdb.org/), TargetScan (http://www.targetscan.org/vert_72/), and microRNA.org (http://www.microrna.org/microrna/home. do). Target genes that appeared in all the three databases were picked out by Cytoscape (Cytoscape 3.7.2, https:// cytoscape.org/download.html) and incorporated into the subsequent analysis.

GO functional enrichment analysis can identify the major biological functions of differentially expressed genes. Kyoto Encyclopedia of Genes and Genomes (KEGG) pathway enrichment can also be utilized to investigate the cellular and organismal functions of tested genes. Therefore, we used GO terms and KEGG pathway analysis to determine the role of differentially expressed mRNAs.

\section{Statistical analyses}

All data were analyzed by SPSS 19.0 (SPSS, IL, USA). Data were presented as mean \pm standard deviation $(\mathrm{SD})$ of three independent experiments. Student's two-tailed $t$-test was used for comparison between two groups, and one-way 
analysis of variance (ANOVA) was used to compare more than two groups. $\mathrm{P}<0.05$ was considered to be statistically significant.

\section{Results}

\section{Dynamic miRNA expressions in buman milk}

The different expression profiles of miRNAs in human colostrum and mature milk were compared using the limma package. There were 782 and 805 known miRNAs identified in colostrum and mature milk, respectively. There were 715 miRNAs in both colostrum and mature milk (Figure 1A). Only a small number of miRNAs were detected exclusively in colostrum or in mature milk: 67 colostrumspecific miRNAs were identified (Table S1), and 89 miRNAs were identified specifically in mature milk (Table $S 2$ ).

Among the 715 miRNAs detected in both colostrum and mature milk, 49 miRNAs were differentially expressed between colostrum and mature milk ( $\mid \log _{2} \mathrm{FCl} \geq 1.58$ with $\mathrm{P}<0.05$ ), including 25 miRNAs that were significantly decreased and 24 miRNAs that were significantly increased in colostrum compared to mature milk (Figure $1 B$ ). Using a fold change $\geq 3$ and $\mathrm{P}<0.05$ as the cutoff threshold, the expressions of 7 miRNAs were significantly altered (Figure 1C). MiR-623 was enriched 10-fold in colostrum compared to in mature milk. In contrast, the levels of miR-30b-5p, miR-885-5p, miR-29c-3p, miR-511-3p, miR-429, and miR-183-5p in colostrum were downregulated compared to those in mature milk. Taken together, these results suggested that there are dynamic changes in the composition of miRNAs in human milk during the human lactation process.

\section{Validation with RT-qPCR}

Next, the expressions of the 7 significantly altered miRNAs were validated in all 18 milk samples using RT-qPCR. After normalization using cel-miR-39 (15), the expression levels of miR-623 in colostrum were $5.052 \pm 0.821$ times higher than those in mature milk $(\mathrm{P}<0.01)$. Meanwhile, the expression levels of miR-30b-5p, miR-885-5p, miR-29c-3p, miR-511-3p, miR-429, and miR-183-5p in colostrum were $0.228 \pm 0.023(\mathrm{P}<0.01), 0.435 \pm 0.025(\mathrm{P}<0.01), 0.434 \pm 0.014$ $(\mathrm{P}<0.01), 0.549 \pm 0.031(\mathrm{P}<0.01), 0.556 \pm 0.013(\mathrm{P}<0.01)$, and $0.722 \pm 0.021(\mathrm{P}<0.01)$ times lower than those in mature milk, respectively (Figure 2). The results of qRT-PCR further confirmed the altered differential expressions of
miRNAs between human colostrum and mature milk.

\section{Predicted target genes of the colostrum-specific miRNAs}

To verify the functions of the identified colostrum-specific miRNAs (Table S1), their target genes were predicted using miRDB, TargetScan, and microRNA.org. The total number of targets predicted by the three databases is 2,134 targets. Using a reliability score (string) $\geq 99 \%$ as a stuff threshold, 10 miRNAs were identified along with 260 targets (Figure 3). This miRNA-mRNA network illustrated the key regulatory functions of the identified miRNAs and their target genes. Briefly, all 10 miRNAs had specific predicted targets. At the same time, different miRNAs have common targets. For instance, miR-3666 and miR-130a-3p had more targets than the other 8 miRNAs. Among these predicted target genes, KCNA4 was the common target gene of 4 miRNAs (miR-3666, miR-130a-3p, miR-6766-3p, and miR-145-5p); UXS1, BTF3L4, and YTHDF2 were the common target genes of 3 miRNAs (miR-3666, miR-6766-3p, and miR$145-5 \mathrm{p})$; SKP1 was the common target gene of miR-3666 and miR-145-5p; NEUROD1 was the common target gene of miR-3666 and miR-378; and CXXC5 was the common target gene of miR-6766-3p and miR-28-3p. These results suggested that the colostrum-specific miRNAs might have unique regulatory functions, but they may also potentially share some similar regulatory functions.

\section{Functional annotation of target genes of the colostrum- specific miRNAs}

Systematic GO analysis of the target genes of the identified colostrum-specific miRNAs indicated that the colostrumspecific miRNAs were involved in the regulation of distinct biological processes. Molecular and functional analysis revealed that most of the targeted mRNAs were related to protein serine/threonine kinase activity (17.67\%), chromatin binding (17.47\%), ubiquitin-protein transferase activity (14.66\%), and nucleotide binding (14.66\%). Other targeted mRNAs were related to ubiquitin protein ligase activity (9.64\%), SH3 domain binding $(7.83 \%)$, and $\beta$-catenin binding (6.02\%). Cellular component analysis showed that mRNA targeted by the miRNAs were mainly in the nucleus $(22.82 \%)$, cytoplasm (22.35), and cytosol $(14.48 \%)$. Others were in the membrane $(9.53 \%)$ and the Golgi apparatus (4.34\%). Biological process analysis showed that mRNA targeted by the miRNAs were involved in signal transduction (24.36\%), positive regulation of GTPase 
A

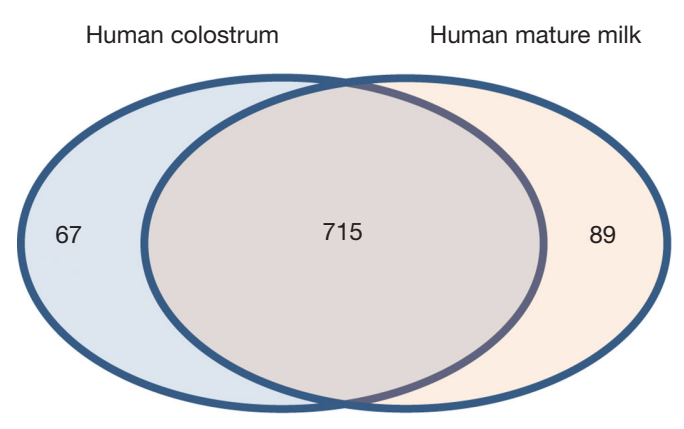

C

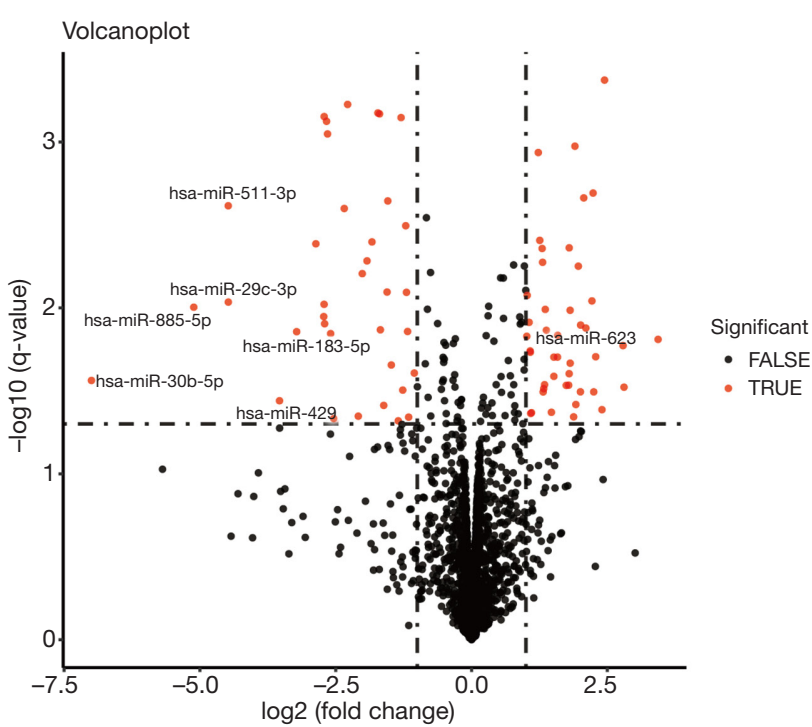

B

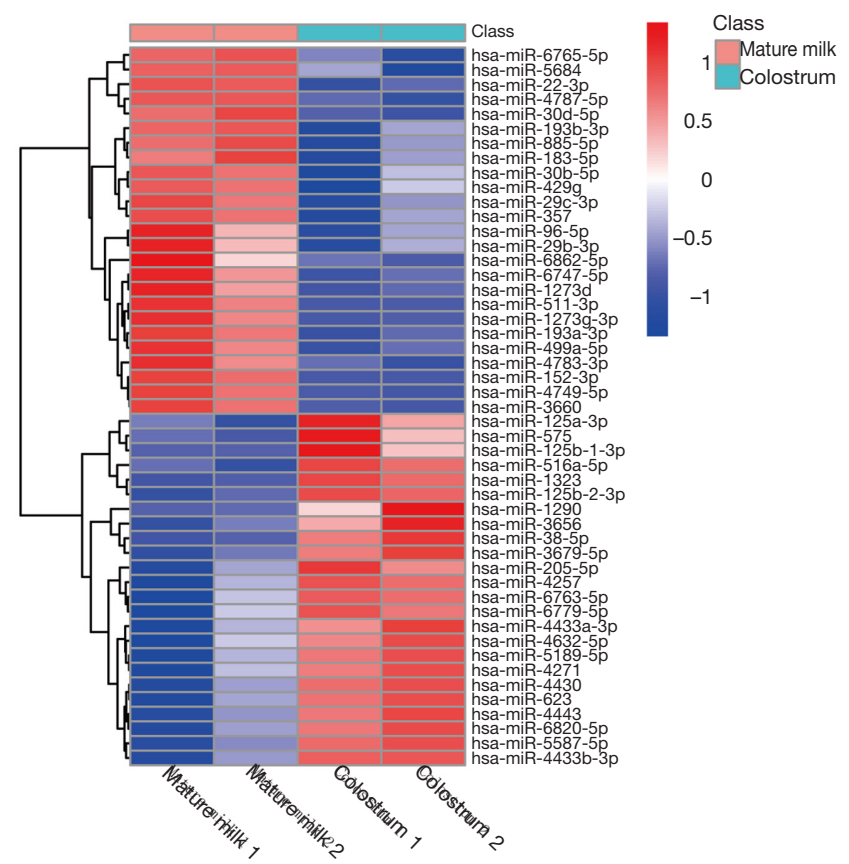

Figure 1 miRNA expressions in human milk. (A) Venn diagram of differentially expressed miRNAs between human colostrum and mature milk. (B) Human colostrum versus human mature milk. Changes in miRNA expression $\log 2 \mathrm{FC}>1.58$, and $\mathrm{P}<0.05$ are illustrated by heat map; blue indicates a relatively low expression and red indicates a relatively high expression. (C) Volcano plot of differentially expressed miRNAs between human colostrum and human mature milk.

activity (12.24\%), protein phosphorylation (10.9\%), intracellular signal transduction $(9.23 \%)$, nervous system development (7.23\%), MAPK cascades (6.79\%), and Wnt signaling (5.34\%) (Figure 4).

In the pathway analysis, the targets of these differentially expressed miRNAs were found to be involved in a wide variety of pathways, including MAPK, PI3K-Akt, endocytic, focal adhesion, and Ras signaling. However, several other relevant pathways were also enriched, such as Rap1, FoxO, Hippo, Wnt, mTOR, and autophagy signaling, as well as longevity regulating pathway (Figure 5). Taken together, these results suggest that human colostrum possesses unique functions compared to mature milk.

\section{Discussion}

Breast milk is the best and most nutritious food for infants. It contains all of the nutrients that babies need for growth and development, and can be easily digested and absorbed. Moreover, since breast milk plays an important 
A

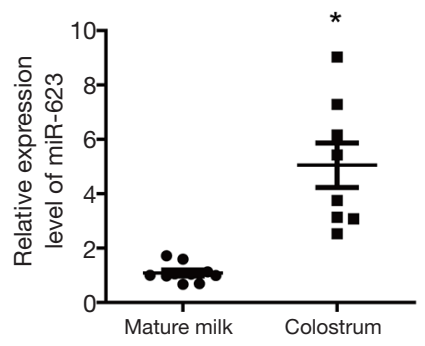

$\mathrm{E}$

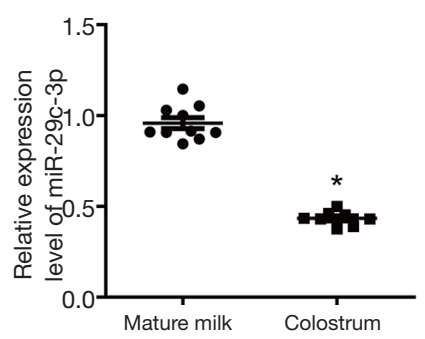

B

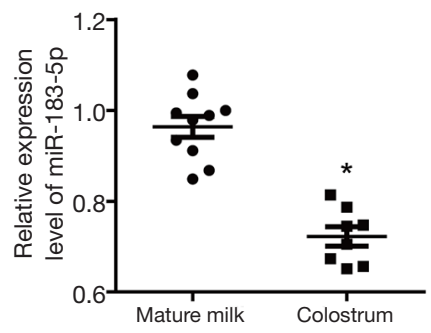

$\mathrm{F}$

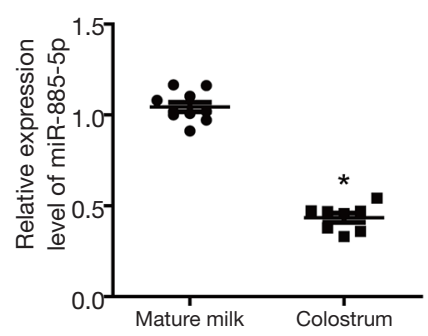

C
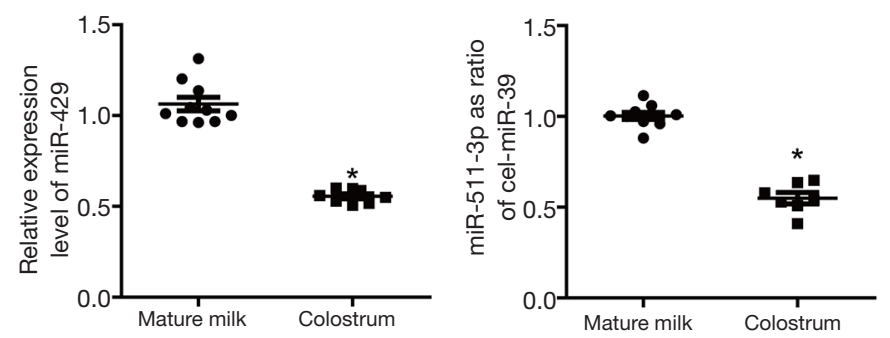

G

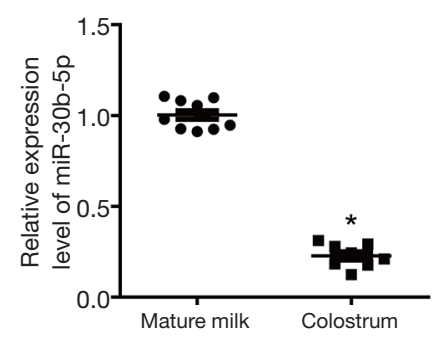

Figure 2 Validation by RT-qPCR. The expression levels of seven randomly selected miRNAs were verified by RT-qPCR, including (A) one up-regulated miRNA (miR-623) and (B,C,D,E,F,G) six down-regulated miRNAs. The relative expression levels of miRNAs were normalized to cel-miR-39 and shown as mean \pm SD.

role in immune system enhancement and systemic disease resistance in infants, breastfeeding can reduce the risks of morbidity and mortality in infants (20).

There are three stages of human lactation: colostrum, transitional milk, and mature milk. Colostrum refers to milk lactated within the 7 days after birth; transitional milk is lactated $7-14$ days after birth; and mature milk is lactated from 2 weeks after birth. The nutritional components of colostrum are obviously different from those of mature milk. Some immunity-related proteins, such as immunoglobulin A, lactoferrin, and lysozyme, are more abundant in colostrum milk than in transitional or mature milk. Many studies have proved that colostrum and its products have many physiological functions, such as regulating immunity, improving gastrointestinal function, promoting growth and development, promoting resistance to pathogenic microorganisms, eliminating fatigue, and delaying aging.

MiRNAs play important roles in the post transcriptional regulation of genes. Multiple miRNAs may regulate the expression of a single specific mRNA, and a single miRNA may also regulate multiple target genes. Moreover, miRNAs have stage-specific and tissue-specific expression patterns. For instance, miR-2285t can target TGFBR1,
RRAS2, RNF111, BMP2, and ACVR2A molecules in TGF- $\beta$ signaling pathway, and many target genes of miR-29b are enriched in PTEN, PI3K/AKT, and JAK/ Stat signaling pathways. MiRNA in breast milk remains stable under severe degradation conditions and can be used as a regulatory mediator in early immune system development and improvement in infants (21). In 2012, Zhou et al. used the Soiexa platform to study the miRNA transcriptome encapsulated by exosomes in human milk. They found 639 mature miRNAs, including 98 immune-related miRNAs (22). Another study showed that the miRNA expression spectrum in pig milk was significantly enriched in immune-related miRNAs. These immune related miRNAs can enhance the immunity of infants (23). Furthermore, miRNAs in breast milk may promote adaptation to early dietary changes through regulating infant metabolism (24).

Our results showed that the composition of miRNAs in human breast milk changes dynamically during lactation process. A total of 782 and 805 known miRNAs were identified in human colostrum and mature milk, respectively; of these miRNAs, 715 were detected in both human colostrum and mature milk. The expression levels of 49 of these 715 miRNAs detected in common were 


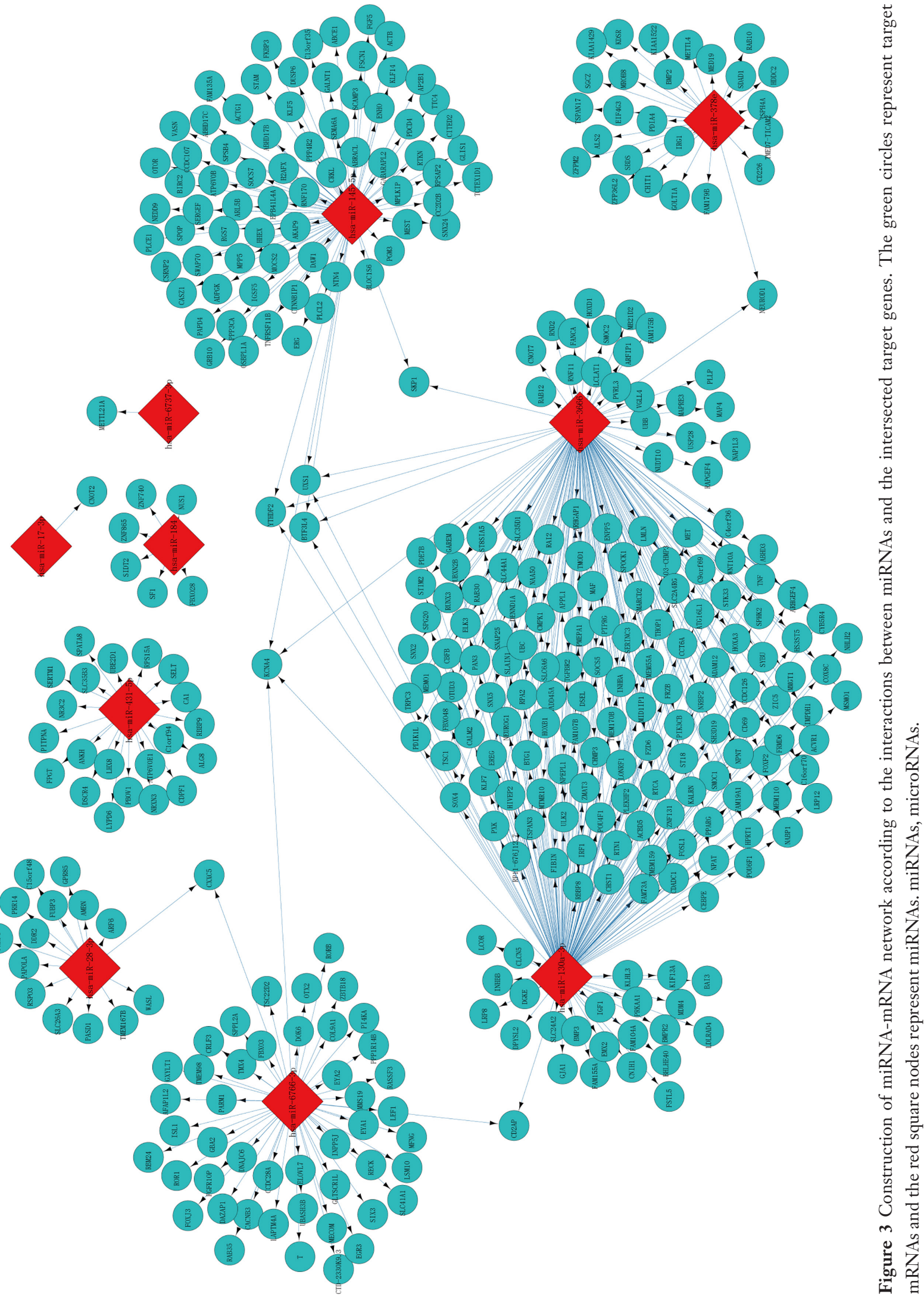




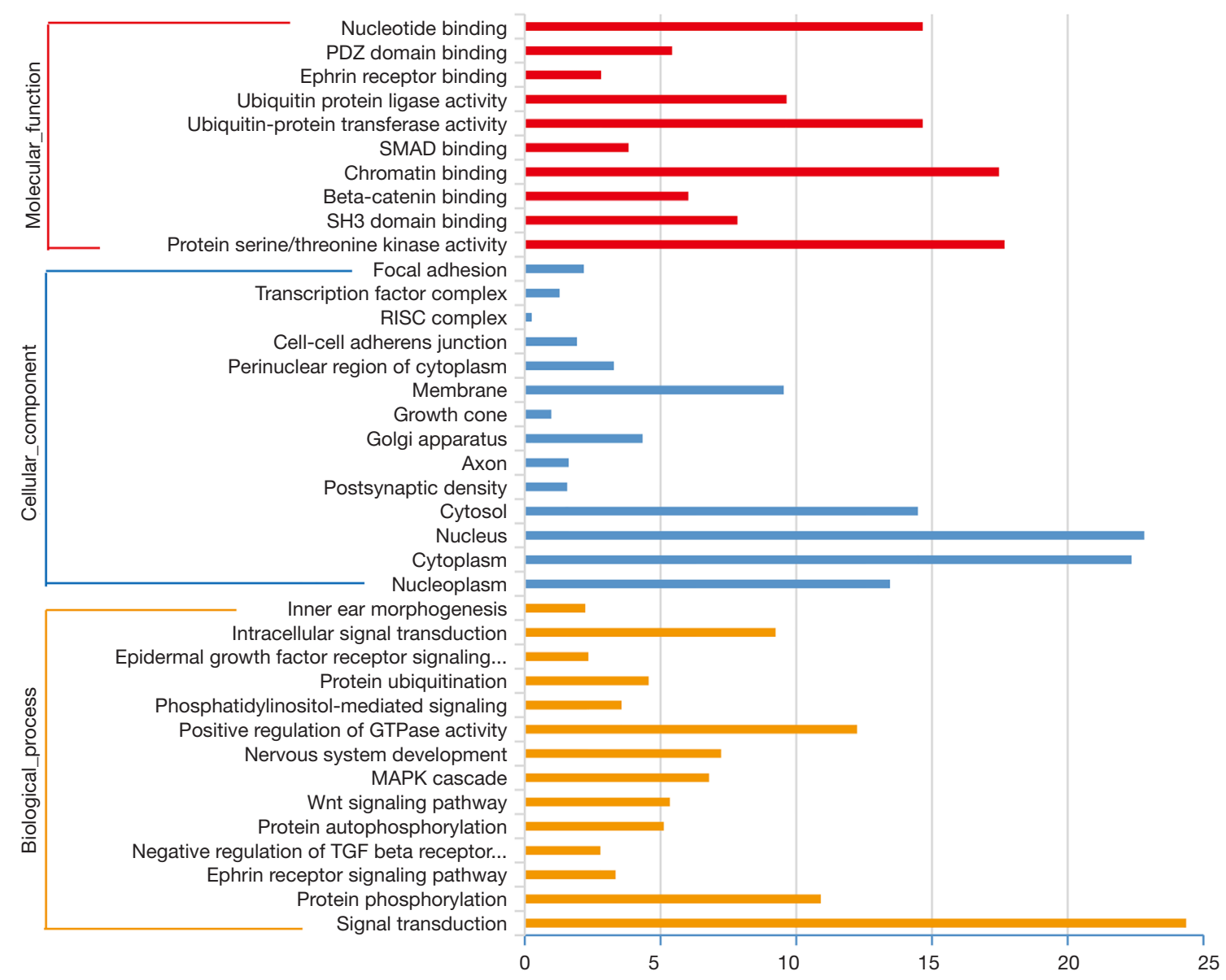

Figure 4 GO analysis of human colostrum-specific miRNA targets. The mRNAs targeted by differentially expressed miRNAs are described in three categories: biological processes, molecular functions, and cellular components. GO, Gene Ontology; miRNAs, microRNAs.

significantly different between colostrum and mature milk. We used qRT-PCR to detect several differentially expressed microRNAs in the collected milk samples. The results of qRT-PCR confirmed the differential expressions of the miRNAs, which were consistent with the results of microarray analysis.

In conclusion, our study identified 67 colostrum-specific miRNAs. The predicted target genes of these miRNAs are closely related to molecular function regulation and binding. KEGG pathway analysis indicated that these targeted mRNAs are closely related to the oxidative stress system, inflammation, development, and longevity. Thus, colostrum might play an important role in the development and even the lifespan of infants. There have been evidence that biologically effective amounts of miRNAs can be absorbed from nutritionally relevant doses of bovine milk, and human gene expression is regulated by physiologic concentrations of milk miRNAs in cell cultures and in vivo $(25,26)$. Moreover, exosomes, a particularly important class of extracellular vesicles, acts as a vehicle to protect and transport labile cargos. For example, milk miRNAs are encapsulated in exosomes to avoid degradation by low $\mathrm{pH}$ in the stomach (27), to resist digestion by enzymes in the gastrointestinal tract (28), and to be specifically transported to receptor cells for local enrichment (29). Taken together, our results indicate that colostrumspecific miRNAs have important functions. These miRNAs could be used as nutritional additives in dairy products to benefit the development and growth of infants and young children. However, expression of miRNA in human milk is influenced by some maternal factors, such as maternal age, dietary habits, and health condition (30), and also by maternal living environment (31). Collections for more human milk samples are needed in future research and also 
A

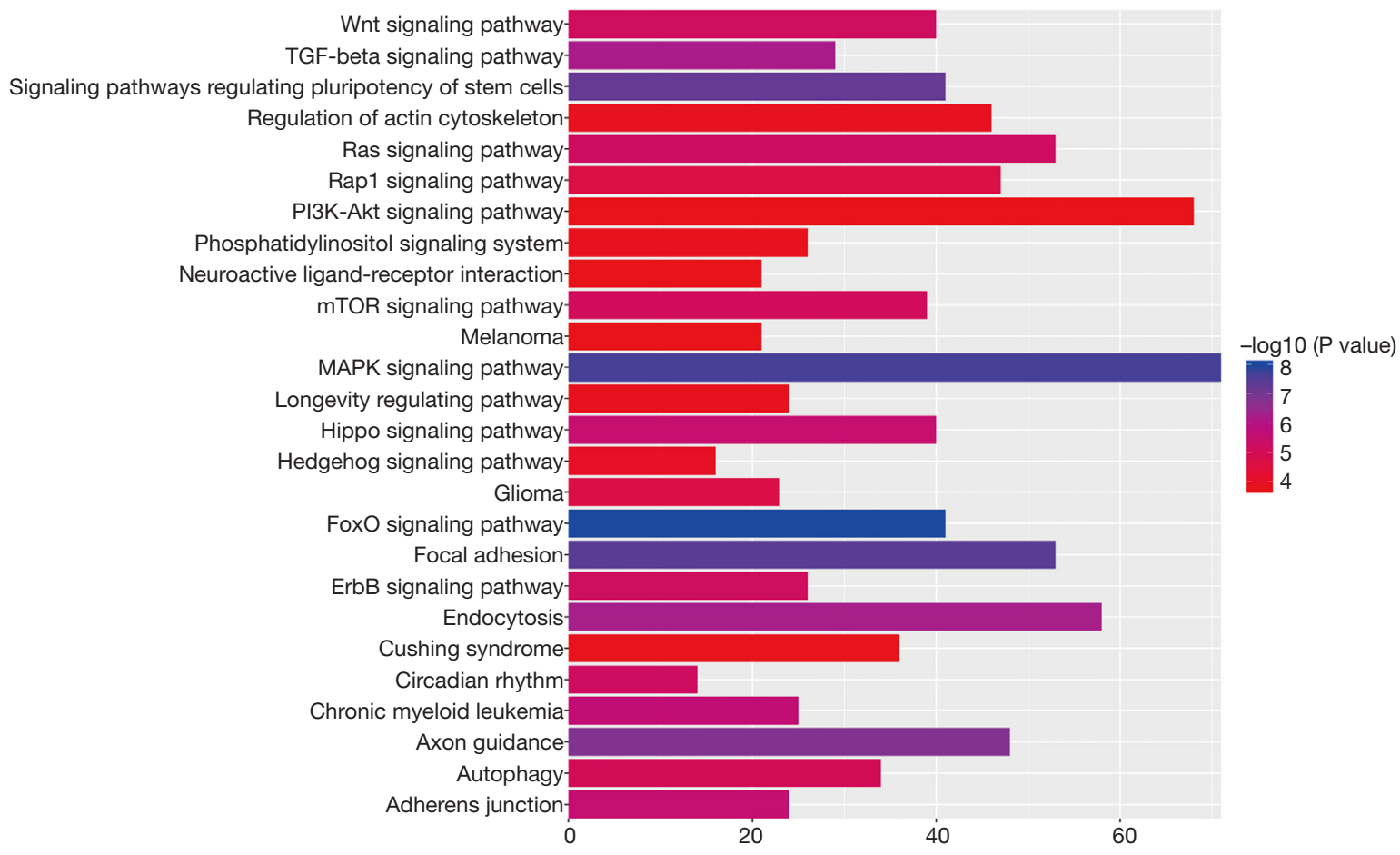

B

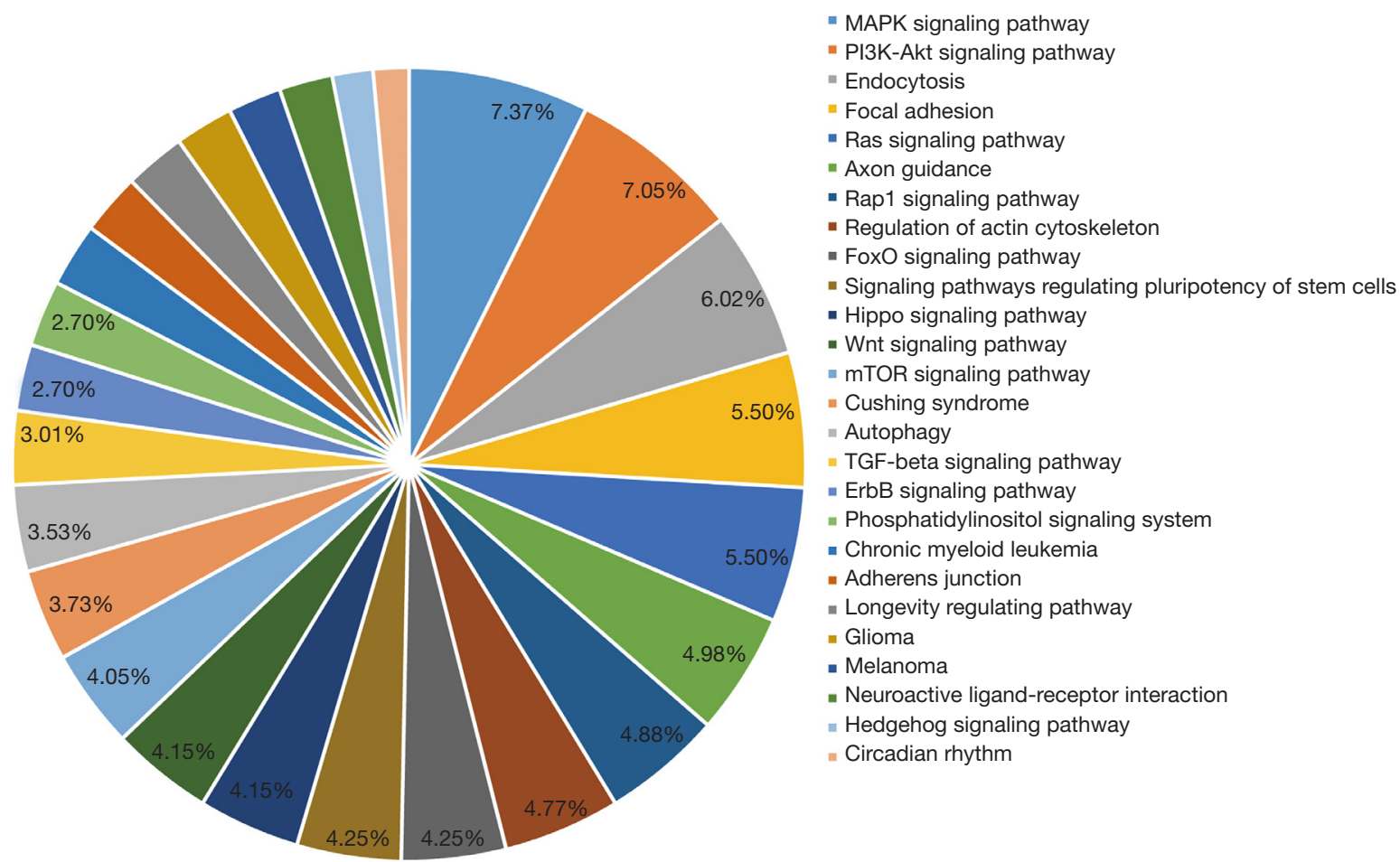

Figure 5 KEGG analysis of human colostrum-specific miRNA targets. (A) shows the enriched pathways and (B) shows the percentage of pathways of the differentially expressed miRNAs using the target mRNAs. KEGG, Kyoto Encyclopedia of Genes and Genomes; miRNAs, microRNAs. 
stratified analysis according to maternal health risk factors are important.

\section{Acknowledgments}

Funding: This work was supported by grants from National Natural Science Foundation of China (81502828, $81872377)$ and Natural Science Foundation of Tianjin City (18JCZDJC35500, 18JCYBJC25600).

\section{Footnote}

Reporting Checklist: The authors have completed the MDAR reporting checklist. Available at http://dx.doi.org/10.21037/ atm-20-5709

Data Sharing Statement: Available at http://dx.doi. org/10.21037/atm-20-5709

Conflicts of Interest: All authors have completed the ICMJE uniform disclosure form (available at http://dx.doi. org/10.21037/atm-20-5709). The authors have no conflicts of interest to declare.

Ethical Statement: The authors are accountable for all aspects of the work in ensuring that questions related to the accuracy or integrity of any part of the work are appropriately investigated and resolved. All procedures performed in this study involving human participants were in accordance with the Declaration of Helsinki (as revised in 2013). This study was approved by Ethics Committee of the second hospital of Tianjin Medical University (No. KY2020K055) and informed consent was taken from all the patients.

Open Access Statement: This is an Open Access article distributed in accordance with the Creative Commons Attribution-NonCommercial-NoDerivs 4.0 International License (CC BY-NC-ND 4.0), which permits the noncommercial replication and distribution of the article with the strict proviso that no changes or edits are made and the original work is properly cited (including links to both the formal publication through the relevant DOI and the license). See: https://creativecommons.org/licenses/by-nc-nd/4.0/.

\section{References}

1. Victora CG, Horta BL, Loret de Mola C, et al. Association between breastfeeding and intelligence, educational attainment, and income at 30 years of age: a prospective birth cohort study from Brazil. Lancet Glob Health 2015;3:e199-205.

2. Molinari CE, Casadio YS, Hartmann BT, et al. Proteome mapping of human skim milk proteins in term and preterm milk. J Proteome Res 2012;11:1696-714.

3. Ha M, Kim VN. Regulation of microRNA biogenesis. Nat Rev Mol Cell Biol 2014;15:509-24.

4. Park NJ, Zhou H, Elashoff D, et al. Salivary microRNA: discovery, characterization, and clinical utility for oral cancer detection. Clin Cancer Res 2009;15:5473-7.

5. Schwarzenbach $\mathrm{H}$. Methods for quantification and characterization of microRNAs in cell-free plasma/serum, normal exosomes and tumor-derived exosomes. Transl Cancer Res 2018;7:S253-63.

6. Zhang C, Fu Q, Ding M, et al. Comprehensive analysis of differentially expressed serum microRNAs in humans responding to Brucella infection. Ann Transl Med 2019;7:301.

7. Mitchell PS, Parkin RK, Kroh EM, et al. Circulating microRNAs as stable blood-based markers for cancer detection. Proc Natl Acad Sci U S A 2008;105:10513-8.

8. Hanke M, Hoefig K, Merz H, et al. A robust methodology to study urine microRNA as tumor marker: microRNA-126 and microRNA-182 are related to urinary bladder cancer. Urol Oncol 2010;28:655-61.

9. Title AC, Denzler R, Stoffel M. Uptake and Function Studies of Maternal Milk-derived MicroRNAs. J Biol Chem 2015;290:23680-91.

10. Zhang L, Huo DX, Chen X, et al. Exogenous plant miR168a specifically targets mammalian LDLRAP1: evidence of cross-kingdom regulation by microRNA. Cell Res 2012;22:107-26.

11. Lang C, Karunairetnam S, Lo KR, et al. Common Variants of the Plant microRNA-168a Exhibit Differing Silencing Efficacy for Human Low-Density Lipoprotein Receptor Adaptor Protein 1 (LDLRAP1). Microrna 2019;8:166-70.

12. Sandhu GK, Milevskiy MJG, Wilson W, et al. Non-coding RNAs in Mammary Gland Development and Disease. Adv Exp Med Biol 2016;886:121-53.

13. Chen $\mathrm{X}$, Gao $\mathrm{C}, \mathrm{Li} \mathrm{H}$, et al. Identification and characterization of microRNAs in raw milk during different periods of lactation, commercial fluid, and powdered milk products. Cell Res 2010;20:1128-37.

14. Playford RJ, Macdonald CE, Johnson WS. Colostrum and milk-derived peptide growth factors for the treatment of gastrointestinal disorders. Am J Clin Nutr 2000;72:5-14. 
15. Dritsakou K, Liosis G, Valsami G, et al. The impact of maternal- and neonatal-associated factors on human milk's macronutrients and energy. J Matern Fetal Neonatal Med 2017;30:1302-8.

16. Castellote C, Casillas R, Ramírez-Santana C, et al. Premature delivery influences the immunological composition of colostrum and transitional and mature human milk. J Nutr 2011;141:1181-7.

17. Ballard O, Morrow AL. Human milk composition: nutrients and bioactive factors. Pediatr Clin North Am 2013;60:49-74.

18. Radmacher PG, Adamkin DH. Fortification of human milk for preterm infants. Semin. Semin Fetal Neonatal Med 2017;22:30-5.

19. Kloten V, Neumann MHD, Di Pasquale F, et al. Multicenter Evaluation of Circulating Plasma MicroRNA Extraction Technologies for the Development of Clinically Feasible Reverse Transcription Quantitative PCR and Next-Generation Sequencing Analytical Work Flows. Clin Chem 2019;65:1132-40.

20. Do DN, Li R, Dudemaine PL, et al. MicroRNA roles in signalling during lactation: an insight from differential expression, time course and pathway analyses of deep sequence data. Sci Rep 2017;7:44605.

21. Izumi $H$, Kosaka N, Shimizu T, et al. Bovine milk contains microRNA and messenger RNA that are stable under degradative conditions. J Dairy Sci 2012;95:4831-41.

22. Zhou Q, Li M, Wang X, et al. Immune-related microRNAs are abundant in breast milk exosomes. Int J Biol Sci 2012;8:118-23.

23. Gu Y, Li M, Wang T, et al. Lactation-related microRNA expression profiles of porcine breast milk exosomes. PLoS One 2012;7:e43691.

Cite this article as: $\mathrm{Wu} \mathrm{F}, \mathrm{Zhi} \mathrm{X}, \mathrm{Xu} \mathrm{R}$, Liang Z, Wang F, Li X, Li Y, Sun B. Exploration of microRNA profiles in human colostrum. Ann Transl Med 2020;8(18):1170. doi: 10.21037/ atm-20-5709
24. Munch EM, Harris RA, Mohammad M, et al. Transcriptome profiling of microRNA by Next-Gen deep sequencing reveals known and novel miRNA species in the lipid fraction of human breast milk. PLoS One 2013;8:e50564.

25. Zempleni J, Aguilar-Lozano A, Sadri M, et al. Biological activities of extracellular vesicles and their cargos from bovine and human milk in humans and implications for infants. J Nutr 2017;147:3-10.

26. Baier SR, Nguyen C, Xie F, et al. MicroRNAs are absorbed in biologically meaningful amounts from nutritionally relevant doses of cow!s milk and affect gene expression in peripheral blood mononuclear cells, HEK-293 kidney cell cultures, and mouse livers. J Nutr 2014;144:1495-500.

27. Izumi H, Kosaka N, Shimizu T, et al. Bovine milk contains microRNA and messenger RNA that are stable under degradative conditions. J Dairy Sci 2012;95:4831-41.

28. Benmoussa A, Lee CHC, Laffont B, et al. Commercial dairy cow milk microRNAs resist digestion under simulated gastrointestinal tract conditions. J Nutr 2016;146:2206-15.

29. Bryniarski K, Ptak W, Jayakumar A, et al. Antigenspecific, antibody-coated, exosome-like nanovesicles deliver suppressor T-cell microRNA-150 to effector T cells to inhibit contact sensitivity. J Allergy Clin Immunol 2013;132:170-81.

30. Xi Y, Jiang X, Li R, et al. The levels of human milk microRNAs and their association with maternal weight characteristics. Eur J Clin Nutr 2016;70:445-9.

31. Li M, Huo X, Davuljigari CB, et al. MicroRNAs and their role in environmental chemical carcinogenesis. Environ Geochem Health 2019;41:225-47. 
Table S1 The colostrum-specific miRNAs identified uniquely in human colostrum

\begin{tabular}{|c|c|c|c|c|c|}
\hline SystematicName & Colostrum1 & Colostrum2 & active_sequence & $\operatorname{chr}$ & mirbase_accession_No \\
\hline hsa-miR-1180-3p & 2.32296 & 2.92182 & ACACACCCACGCG & chr17 & MIMAT0005825 \\
\hline hsa-miR-1199-5p & 2.54510 & 3.10266 & CTGCGCGGCCC & chr19 & MIMAT0031119 \\
\hline hsa-miR-1229-3p & 2.62540 & 3.07745 & CTGTGGGAGGGC & chr5 & MIMAT0005584 \\
\hline hsa-miR-1237-3p & 2.55619 & 2.72214 & CTGGGGGACGG & chr11 & MIMAT0005592 \\
\hline hsa-miR-1254 & 2.20128 & 3.20950 & ACTGCAGGCTCCAGC & chr10 & MIMAT0005905 \\
\hline hsa-miR-1266-3p & 2.59962 & 2.94387 & TCCCTCAGGGCATAGA & chr15 & MIMAT0026742 \\
\hline hsa-miR-1295a & 2.11806 & 4.17877 & TCACCCAGATCTGCG & chr1 & MIMAT0005885 \\
\hline hsa-miR-1304-3p & 2.98629 & 2.68252 & GGGGTTCGAGGCT & chr11 & MIMAT0022720 \\
\hline hsa-miR-130a-3p & 3.67398 & 2.18873 & ATGCCCTTTTAACATTGCA & chr11 & MIMAT0000425 \\
\hline hsa-miR-145-5p & 2.71837 & 2.20168 & AGGGATTCCTGGGAAAAC & chr5 & MIMAT0000437 \\
\hline hsa-miR-17-3p & 2.50705 & 2.73279 & CTACAAGTGCCTTCAC & chr13 & MIMAT0000071 \\
\hline hsa-miR-1825 & 3.02946 & 2.38080 & GGAGAGGAGGGCAC & chr20 & MIMAT0006765 \\
\hline hsa-miR-184 & 2.35008 & 3.04543 & ACCCTTATCAGTTCTCCGTCCA & chr15 & MIMAT0000454 \\
\hline hsa-miR-2277-3p & 1.97716 & 2.90604 & GAGCCAGGCAGGG & chr5 & MIMAT0011777 \\
\hline hsa-miR-28-3p & 3.14458 & 2.74099 & TCCAGGAGCTCACA & chr3 & MIMAT0004502 \\
\hline hsa-miR-298 & 2.05152 & 2.66207 & TGGGAGAACCTCCCTG & chr20 & MIMAT0004901 \\
\hline hsa-miR-3130-3p & 2.30457 & 2.63398 & TTACCCAGTCTCCGG & chr2 & MIMAT0014994 \\
\hline hsa-miR-3163 & 2.21398 & 3.49302 & GTCTTACTGCCCTCATT & chr11 & MIMAT0015037 \\
\hline hsa-miR-3170 & 2.07935 & 2.95889 & ACTGTCTGTCTCAGAACC & chr13 & MIMAT0015045 \\
\hline hsa-miR-3180-5p & 2.86044 & 2.93355 & CGACGTGGGGCG & chr16 & MIMAT0015057 \\
\hline hsa-miR-3182 & 2.64436 & 1.98775 & GACTACACTACAGAAGC & chr16 & MIMAT0015062 \\
\hline hsa-miR-3187-5p & 2.16980 & 2.88324 & CCTTCAGCCACACG & chr19 & MIMAT0019216 \\
\hline hsa-miR-3651 & 2.76310 & 1.98912 & TCATGTACCAGCGACC & chr9 & MIMAT0018071 \\
\hline hsa-miR-3653-3p & 1.99244 & 2.84147 & СTTCAGTCAACTTCTTAG & chr22 & MIMAT0018073 \\
\hline hsa-miR-3666 & 2.14131 & 2.86701 & TCGGCATCTACACTTGC & chr7 & MIMAT0018088 \\
\hline hsa-miR-3679-3p & 2.11051 & 2.43942 & GATGAAGATTACTGGGGG & chr2 & MIMAT0018105 \\
\hline hsa-miR-3689a-5p & 3.54821 & 3.36309 & TCCCAGGAACCATGAT & chr9 & MIMAT0018117 \\
\hline hsa-miR-3689f & 3.92421 & 3.86609 & TCCCAGGAAGCACG & chr9 & MIMAT0019010 \\
\hline hsa-miR-378e & 2.29603 & 2.43965 & TCCTGACTCCAAGTCC & chr5 & MIMAT0018927 \\
\hline hsa-miR-3934-3p & 3.11223 & 3.03074 & TCCCAGCTGTGCAAC & chr6 & MIMAT0022975 \\
\hline hsa-miR-4265 & 3.97453 & 2.44913 & CCCAGAGCTGAGCC & chr2 & MIMAT0016891 \\
\hline hsa-miR-4282 & 3.95189 & 3.86366 & TCCTGGATGCAAATTTTA & chr6 & MIMAT0016912 \\
\hline hsa-miR-431-5p & 2.19106 & 2.57381 & TGCATGACGGCCTGC & chr14 & MIMAT0001625 \\
\hline hsa-miR-4446-3p & 2.13045 & 3.12006 & ACCCATGTCACTGCC & chr3 & MIMAT0018965 \\
\hline hsa-miR-4489 & 2.39445 & 3.03111 & CGTCCTGCATCACTAG & chr11 & MIMAT0019023 \\
\hline hsa-miR-4492 & 2.15679 & 2.80300 & GGCGCGCGCC & chr11 & MIMAT0019027 \\
\hline hsa-miR-4510 & 2.59543 & 2.87916 & ААССАТАСАТССТАСТССC & chr15 & MIMAT0019047 \\
\hline hsa-miR-4533 & 2.17802 & 2.77478 & AGCGTCCGGCAAC & chr20 & MIMAT0019072 \\
\hline hsa-miR-4636 & 1.96493 & 4.79457 & CTAAAGGCTTGAACACG & chr5 & MIMAT0019693 \\
\hline hsa-miR-4715-5p & 2.42423 & 3.20679 & ССАССTTAACTGCAGC & chr15 & MIMAT0019824 \\
\hline hsa-miR-4717-3p & 2.27863 & 2.40895 & AGGCCACAGCCACC & chr16 & MIMAT0019830 \\
\hline hsa-miR-4731-3p & 2.40021 & 2.70453 & AGTGTTGGGGGCCA & chr17 & MIMAT0019854 \\
\hline hsa-miR-4773 & 2.43453 & 3.21978 & GCCTTTCTATGCTCCTG & chr2 & MIMAT0019928 \\
\hline hsa-miR-509-3-5p & 1.87485 & 2.65251 & CATGATTGCCACGTCTG & $\operatorname{chr} \mathrm{x}$ & MIMAT0004975 \\
\hline hsa-miR-509-5p & 2.01383 & 3.08984 & TGATTGCCACTGTCTGC & $\operatorname{chr} x$ & MIMAT0004779 \\
\hline hsa-miR-510-3p & 2.39686 & 2.80545 & TCCACTCTTAGAGGTTTC & chrX & MIMAT0026613 \\
\hline hsa-miR-513c-3p & 2.27689 & 2.71956 & TCTTCTCAGAAAGGTGAA & chrX & MIMAT0022728 \\
\hline hsa-miR-516a-3p & 2.20430 & 2.50209 & АСССTCTGAAAGGAAGCA & chr19 & MIMAT0006778 \\
\hline hsa-miR-598-5p & 1.89566 & 3.08960 & GCTCACACCATCGG & chr8 & MIMAT0026620 \\
\hline hsa-miR-639 & 1.88307 & 2.10263 & ACAGCGCTCGCAACCGC & chr19 & MIMAT0003309 \\
\hline hsa-miR-658 & 2.13478 & 2.09188 & ACCAACGGACCTACTTCCCT & chr22 & MIMAT0003336 \\
\hline hsa-miR-663b & 1.97456 & 2.87451 & СCTCAGGCACGGC & chr2 & MIMAT0005867 \\
\hline hsa-miR-664a-3p & 3.06870 & 2.17013 & TGTAGGCTGGGGATAAA & chr1 & MIMAT0005949 \\
\hline hsa-miR-6722-5p & 2.01272 & 2.81332 & GCATGTGGTCGGGT & chr9 & MIMAT0025853 \\
\hline hsa-miR-6737-3p & 2.89093 & 2.48512 & CTGGGTAGGGGTGA & chr1 & MIMAT0027376 \\
\hline hsa-miR-6752-3p & 2.33123 & 2.44844 & CTGGGAGTATGGGGG & chr11 & MIMAT0027405 \\
\hline hsa-miR-6763-3p & 2.44360 & 2.85405 & CTGGGGGCAGAGG & chr12 & MIMAT0027427 \\
\hline hsa-miR-6766-3p & 2.53711 & 2.83010 & TGAGGGTGGGGGAA & chr15 & MIMAT0027433 \\
\hline hsa-miR-6771-5p & 2.46932 & 2.96786 & GCCTGGCCCATGC & chr16 & MIMAT0027442 \\
\hline hsa-miR-6795-5p & 2.12781 & 3.19112 & ACAGCCTCTCATCCTG & chr19 & MIMAT0027490 \\
\hline hsa-miR-6871-5p & 2.26893 & 3.55816 & GCAACCACCCCGA & chr20 & MIMAT0027642 \\
\hline hsa-miR-7157-5p & 2.16873 & 2.70452 & TCTCTGGTGCCAATGA & chr2 & MIMAT0028224 \\
\hline hsa-miR-7854-3p & 2.14090 & 2.81021 & TTCCCATCTGCGGTC & chr16 & MIMАТ0030429 \\
\hline hsa-miR-8052 & 2.29522 & 2.84037 & GCTCATGCCCTCTACA & chr11 & MIMAT0030979 \\
\hline hsa-miR-8075 & 1.91768 & 3.67316 & CAGACCCGACATCTG & chr13 & MIMAT0031002 \\
\hline hsa-miR-8078 & 1.94227 & 2.57651 & GAGTCTCTCACCGGG & chr18 & MIMAT0031005 \\
\hline hsa-miR-873-3p & 2.52308 & 2.71775 & TCCCGGGAACTCATC & chr9 & MIMAT0022717 \\
\hline
\end{tabular}


Table S2 The miRNAs identified specifically in human mature milk

\begin{tabular}{|c|c|c|c|c|c|}
\hline SystematicName & Mature1 & Mature2 & active_sequence & chr & mirbase_accession_No \\
\hline hsa-miR-10a-3p & 1.98751 & 2.63176 & TATTCCCCTAGATACGAA & chr17 & MIMAT0004555 \\
\hline hsa-miR-1203 & 2.24244 & 2.85905 & GAGCTGCATCCTGGC & chr17 & MIMAT0005866 \\
\hline hsa-miR-1207-3p & 2.25352 & 2.56912 & GAAATGAGGGCCAGC & chr8 & MIMAT0005872 \\
\hline hsa-miR-1237-5p & 2.33949 & 2.57669 & CGCGCTTCGGCC & chr11 & MIMAT0022946 \\
\hline hsa-miR-1261 & 2.65172 & 2.48569 & AAGCCAAAGCCTTATCC & chr11 & MIMAT0005913 \\
\hline hsa-miR-1273d & 4.68993 & 5.83660 & ACTGCAGCCTCAACC & chr1 & MIMAT0015090 \\
\hline hsa-miR-1273g-5p & 2.38639 & 3.44264 & ACTTACTGCAGCCTCAA & chr1 & MIMAT0020602 \\
\hline hsa-miR-1273h-3p & 2.62297 & 2.54658 & GCCTGGGAGGTCG & chr16 & MIMAT0030416 \\
\hline hsa-miR-1273h-5p & 2.07145 & 2.68090 & ACTGCAGCCTTGACC & chr16 & MIMAT0030415 \\
\hline hsa-miR-128-3p & 2.46776 & 2.53820 & AAAGAGACCGGTTCACTGT & chr3 & MIMAT0000424 \\
\hline hsa-miR-1285-3p & 1.90388 & 2.40571 & AGGTCTCACTTTGTTGC & chr7 & MIMAT0005876 \\
\hline hsa-miR-1287-5p & 2.65848 & 2.65860 & GACTCGAACCACTGAT & chr10 & MIMAT0005878 \\
\hline hsa-miR-129-5p & 2.64066 & 2.11382 & GCAAGCCCAGACCGC & chr7 & MIMAT0000242 \\
\hline hsa-miR-138-5p & 2.17710 & 3.01523 & CGGCCTGATTCACA & chr3 & MIMAT0000430 \\
\hline hsa-miR-150-5p & 2.01261 & 2.34502 & CACTGGTACAAGGGTGG & chr19 & MIMAT0000451 \\
\hline hsa-miR-152-3p & 3.64082 & 3.90271 & CCAAGTTCTGTCATGC & chr17 & MIMAT0000438 \\
\hline hsa-miR-182-5p & 2.19165 & 2.30981 & AGTGTGAGTTCTACCAT & chr7 & MIMAT0000259 \\
\hline hsa-miR-183-3p & 2.39574 & 2.31177 & TATGGCCCTTCGGT & chr7 & MIMAT0004560 \\
\hline hsa-miR-185-5p & 2.74797 & 3.14207 & TCAGGAACTGCCTTTCT & chr22 & MIMAT0000455 \\
\hline hsa-miR-18a-5p & 2.12542 & 2.38964 & СTATCTGCACTAGATGCA & chr13 & MIMAT0000072 \\
\hline hsa-miR-1910-3p & 2.52924 & 2.68037 & TGTCATCCTGCTTCTGC & chr16 & MIMAT0026917 \\
\hline hsa-miR-1972 & 2.53313 & 2.76746 & TGAGCCACTGTGCC & chr16 & MIMAT0009447 \\
\hline hsa-miR-1973 & 2.71066 & 2.94558 & TATGCTACCTTTGCACG & chr4 & MIMAT0009448 \\
\hline hsa-miR-199a-3p & 2.33512 & 2.71778 & TAACCAATGTGCAGACTACT & chr19 & MIMAT0000232 \\
\hline hsa-miR-210-5p & 2.91142 & 2.65243 & CAGTGTGCGGTGGG & chr11 & MIMAT0026475 \\
\hline hsa-miR-22-5p & 3.42928 & 2.99324 & TAAAGCTTGCCACTGAAG & chr17 & MIMAT0004495 \\
\hline hsa-miR-29c-5p & 3.11572 & 4.41128 & GAACACCAGGAGAAATCGGT & chr1 & MIMAT0004673 \\
\hline hsa-miR-30e-3p & 2.62255 & 2.72617 & GCTGTAAACATCCGACTG & chr1 & MIMAT0000693 \\
\hline hsa-miR-3150b-5p & 2.82997 & 2.15772 & GCTGGGGAGATCCTC & chr8 & MIMAT0019226 \\
\hline hsa-miR-3159 & 2.16115 & 3.25306 & GTGGCCGACACTTG & chr11 & MIMAT0015033 \\
\hline hsa-miR-3164 & 2.30669 & 2.91856 & CGCCATTTCCCTTAAA & chr11 & MIMAT0015038 \\
\hline hsa-miR-3174 & 2.16155 & 2.95890 & GGCTCTGCATCTCTAAC & chr15 & MIMAT0015051 \\
\hline hsa-miR-3177-3p & 2.82184 & 2.49063 & ACGTGTCCCCAGTGC & chr16 & MIMAT0015054 \\
\hline hsa-miR-324-5p & 2.50719 & 2.63198 & ACACCAATGCCCTAGGG & chr17 & MIMAT0000761 \\
\hline hsa-miR-338-3p & 2.13770 & 2.52962 & CAACAAAATCACTGATGCTGG & chr17 & MIMATO000763 \\
\hline hsa-miR-339-3p & 2.58758 & 3.10585 & CGGCTCTGTCGTCG & $\mathrm{chr} 7$ & MIMAT0004702 \\
\hline hsa-miR-340-5p & 2.43818 & 3.20849 & ААTCAGTCTCATTGCTTTA & chr5 & MIMAT0004692 \\
\hline hsa-miR-3619-3p & 2.01330 & 2.78057 & CCACAGCAGGCAGG & chr22 & MIMAT0019219 \\
\hline hsa-miR-365b-5p & 2.95847 & 2.68694 & ACAGCTGCCCCTGA & chr17 & MIMAT0022833 \\
\hline hsa-miR-3935 & 2.42008 & 2.59386 & GTGGCTGGTGCTCG & chr16 & MIMAT0018350 \\
\hline hsa-miR-4267 & 3.09477 & 3.92122 & GTGCCACCGAGCT & chr2 & MIMAT0016893 \\
\hline hsa-miR-4284 & 3.91543 & 2.18863 & ATGGGGTGATGTGAGC & $\mathrm{chr} 7$ & MIMAT0016915 \\
\hline hsa-miR-4291 & 3.18635 & 4.27236 & AGCTGTTCCTGCTGAA & chr9 & MIMAT0016922 \\
\hline hsa-miR-4418 & 2.50637 & 2.67114 & CTGCTGAGTCCTGCA & chr1 & MIMAT0018930 \\
\hline hsa-miR-4421 & 2.09017 & 2.85002 & TAGCTCCTTTCCACAGA & chr1 & MIMAT0018934 \\
\hline hsa-miR-4458 & 2.36574 & 2.61123 & ПТПТССАСАССТАССТ & chr5 & MIMAT0018980 \\
\hline hsa-miR-4475 & 2.03341 & 2.51302 & ATAATGAATGCTTGGTCCC & chr9 & MIMAT0019002 \\
\hline hsa-miR-4479 & 2.60301 & 2.53507 & CTGCTCCGAGCACG & chr9 & MIMAT0019011 \\
\hline hsa-miR-4482-3p & 2.46688 & 3.25644 & GAGCCCCACTGAGA & chr10 & MIMAT0020958 \\
\hline hsa-miR-4494 & 2.51388 & 3.01822 & CCTCTGGTCAGCCA & chr12 & MIMAT0019029 \\
\hline hsa-miR-4526 & 2.09555 & 2.37480 & AGCGGCCAGCCC & chr18 & MIMAT0019065 \\
\hline hsa-miR-4638-5p & 2.05626 & 2.75095 & ACTTGTCCACCGCAG & chr5 & MIMAT0019695 \\
\hline hsa-miR-4657 & 2.36918 & 3.14834 & ATGCCTCAGACCACTT & chr7 & MIMAT0019724 \\
\hline hsa-miR-4694-3p & 2.14011 & 2.68527 & AGGTGTTATCCTGTCCA & chr11 & MIMAT0019787 \\
\hline hsa-miR-4725-5p & 2.83625 & 2.59880 & GGTGGGAAGGCTGC & chr17 & MIMAT0019843 \\
\hline hsa-miR-4727-3p & 2.18564 & 2.82298 & GAATCTGCCAGCTTCC & chr17 & MIMAT0019848 \\
\hline hsa-miR-4746-5p & 2.50482 & 2.71830 & TCTGCAGGTTCTCCTG & chr19 & MIMAT0019880 \\
\hline hsa-miR-4768-3p & 2.30560 & 2.87099 & АТTCTCTCTGGATCTCCT & $\operatorname{chr} x$ & MIMAT0019921 \\
\hline hsa-miR-484 & 3.18353 & 3.33444 & ATCGGGAGGGGACTGA & chr16 & MIMATO002174 \\
\hline hsa-miR-499a-5p & 3.89492 & 4.44918 & AAACATCACTGCAAGTCTTAA & chr20 & MIMAT0002870 \\
\hline hsa-miR-5003-5p & 2.48713 & 2.62131 & TCTACCCTGCAAGGTT & chr5 & MIMAT0021025 \\
\hline hsa-miR-501-3p & 2.07789 & 2.63682 & AGAATCCTTGCCCGGG & $\operatorname{chr} X$ & MIMAT0004774 \\
\hline hsa-miR-5093 & 2.62512 & 2.40514 & GCTCCTAGCCAGCC & chr16 & MIMAT0021085 \\
\hline hsa-miR-5096 & 2.05944 & 3.04100 & GCCTGACCAACATGG & chr4 & MIMAT0020603 \\
\hline hsa-miR-511-3p & 5.81124 & 6.95428 & TCTGTCПTाTGCTACACA & chr10 & MIMAT0026606 \\
\hline hsa-miR-517a-3p & 3.93271 & 1.99377 & ACACTCTAAAGGGATGCAC & chr19 & MIMAT0002852 \\
\hline hsa-miR-517c-3p & 2.82146 & 2.00449 & ACACTCTAAAAGGATGCAC & chr19 & MIMAT0002866 \\
\hline hsa-miR-518a-5p & 2.04188 & 2.55783 & GAAAGGGCTTCCCTT & chr19 & MIMAT0005457 \\
\hline hsa-miR-522-3p & 3.31805 & 1.92379 & ACACTCTAAAGGGAACCATT & chr19 & MIMAT0002868 \\
\hline hsa-miR-541-3p & 2.21166 & 2.54955 & AGTCCAGATTCTGTGCCC & chr14 & MIMAT0004920 \\
\hline hsa-miR-574-3p & 3.71477 & 3.03704 & TGTGGGTGTGTGCATG & chr4 & MIMAT0003239 \\
\hline hsa-miR-593-5p & 1.85946 & 2.61223 & GCTGAGCAATGCCTG & chr7 & MIMAT0003261 \\
\hline hsa-miR-629-3p & 2.27893 & 2.78905 & GCTGGGCTTACGTTGG & chr15 & MIMAT0003298 \\
\hline hsa-miR-6508-5p & 2.91166 & 2.97465 & GGTGGGTCATGCATT & chr21 & MIMAT0025472 \\
\hline hsa-miR-652-3p & 2.41564 & 2.60999 & CACAACCCTAGTGGC & $\operatorname{chr} x$ & MIMAT0003322 \\
\hline hsa-miR-664a-5p & 2.34364 & 2.45058 & ATCCAATCATTTTCCCTAGC & chr1 & MIMAT0005948 \\
\hline hsa-miR-664b-5p & 2.13124 & 3.56128 & TACCCAATCATCTCCCT & $\operatorname{chr} x$ & MIMAT0022271 \\
\hline hsa-miR-6726-5p & 2.91249 & 2.72714 & АCCTGCAGACCCCA & chr1 & MIMAT0027353 \\
\hline hsa-miR-6753-3p & 2.84822 & 3.51372 & GTGCCAGGGCAGA & chr11 & MIMAT0027407 \\
\hline hsa-miR-6813-3p & 2.63163 & 2.32766 & CTGGGGAGAGGGG & chr20 & MIMAT0027527 \\
\hline hsa-miR-6855-5p & 1.93228 & 2.44027 & GCAATGTCTGCACCC & chr9 & MIMAT0027610 \\
\hline hsa-miR-6868-5p & 1.99168 & 2.49615 & GCTGCTTCAGTGTTCT & chr17 & MIMAT0027636 \\
\hline hsa-miR-6884-5p & 4.09949 & 3.50563 & CAACATCACCTTCTCAG & chr17 & MIMAT0027668 \\
\hline hsa-miR-7114-3p & 2.68504 & 2.54284 & CTGGTGGAGAGGGG & chr9 & MIMAT0028126 \\
\hline hsa-miR-766-3p & 3.02450 & 2.87476 & GCTGAGGCTGTGGGGCT & $\operatorname{chr} x$ & MIMAT0003888 \\
\hline hsa-miR-7856-5p & 3.52941 & 3.85569 & GATCCCTCAGTGTCCT & chr1 & MIMAT0030431 \\
\hline hsa-miR-8077 & 2.31582 & 2.82875 & GGAGTCAGAACCCCA & chr19 & MIMAT0031004 \\
\hline hsa-miR-885-3p & 2.12362 & 2.12247 & TATCCACTACACCCCG & chr3 & MIMAT0004948 \\
\hline nsa-miR-921 & 2.76696 & 2.55530 & GAATCCTGGTTCTGTCC & chr1 & MIMAT0004971 \\
\hline hsa-miR-96-5p & 3.52452 & 4.40404 & AGCAAAAATGTGCTAGTGCCAA & chr7 & MIMAT0000095 \\
\hline
\end{tabular}

